\title{
On $\left(\mathrm{r}^{*} \mathrm{~g}^{*}\right)^{*}$ Closed Sets in Topological Spaces
}

\author{
N. Meenakumari ${ }^{1}$, T. Indira ${ }^{2}$ \\ 1,2PG and Research Department of Mathematics, Seethalakshmi Ramaswamy College (Autonomous), Trichirapalli-620002, India
}

\begin{abstract}
The aim of this paper is to introduce a new class of sets namely ( $\left.r * g^{*}\right) *$ closed sets in topological spaces. This class was obtained by generalizing closed sets via $r * g *$ open sets which was introduced by N.Meenakumari and T.Indira[16].This new class falls strictly between the class of closed sets and rg closed sets.
\end{abstract}

\section{Mathematics Subject Classification: 54A05}

Keywords: $\left(\mathrm{r}^{*} \mathrm{~g}^{*}\right) *$ closed sets. $\left(\mathrm{r}^{*} \mathrm{~g}\right)^{*}$ open sets

\section{Introduction}

In 1970, Levine [12] introduced the concept of generalized closed set in the topological spaces. Many mathematicians started generalizing closed sets in recent years. In 1993, N. Palaniappan and K. Chandrasekhara Rao [22] introduced regular generalized closed (rg-closed) sets. M. K. R. S. Veerakumar introduced $\mathrm{g} *$ closed sets [26], g\#closed sets [25 ] in topological spaces. The aim of this paper is to introduce a new class of sets namely $\left(\mathrm{r}^{*} \mathrm{~g}^{*}\right)^{*}$ closed sets in topological spaces and study some basic properties.

\section{Preliminaries}

Definition:2.1: A subset A of a space $\mathrm{X}$ is called

(1) a preopen set if $\mathrm{A} \subseteq \operatorname{int}(\mathrm{cl}(\mathrm{A}))$ and a preclosed set if $\operatorname{cl}(\operatorname{int}(\mathrm{A})) \subseteq \mathrm{A}$.

(2) a semi-open set if $\mathrm{A} \subseteq \mathrm{cl}(\operatorname{int}(\mathrm{A}))$ and a semi-closed set if (int(cl(A) $\subseteq$ A.

(3) an $\alpha$-open set if $\mathrm{A} \subseteq \operatorname{int}(\mathrm{cl}(\operatorname{int}(\mathrm{A})))$ and a $\alpha$-closed set if $\operatorname{cl}(\operatorname{int}(\mathrm{cl}(\mathrm{A}))) \subseteq \mathrm{A}$.

(4) A semi-preopen set ( $\beta$-open) if $\mathrm{A} \subseteq \operatorname{cl}(\operatorname{int}(\operatorname{cl}(\mathrm{A})))$ and a semi- preclosed set $(\beta$-closed $)$ if int $(\operatorname{cl}(\operatorname{int}(\mathrm{A}))) \subseteq \mathrm{A}$.

Definition:2.2: A subset A of a space $\mathrm{X}$ is called

1. A Regular closed[24] if $\operatorname{cl}(\operatorname{int}(\mathrm{A}))=\mathrm{A}$.

2. A generalized closed (g closed) [12] set if $\operatorname{cl}(\mathrm{A}) \subseteq \mathrm{U}$ whenever $\mathrm{A} \subseteq \mathrm{U}$ and $\mathrm{U}$ is open.

3. A Regular generalized closed (rg-closed) [22 ] set if $\mathrm{cl}(\mathrm{A}) \subseteq \mathrm{U}$ whenever $\mathrm{A} \subseteq \mathrm{U}$ and $\mathrm{U}$ is regular open.

4. An $\alpha$-generalized closed ( $\alpha$ g- closed) [8] set if $\alpha \operatorname{cl}(\mathrm{A}) \subseteq \mathrm{U}$ whenever $\mathrm{A} \subseteq \mathrm{U}$ and $\mathrm{U}$ is open.

5. A semi generalized closed (briefly sg - closed) [6] if $\operatorname{scl}(\mathrm{A}) \subseteq \mathrm{U}$ whenever $(\mathrm{A}) \subseteq \mathrm{U}$ and $\mathrm{U}$ is semiopen in $\mathrm{X}$.
6. A generalized semi closed (briefly gs - closed) [2] if $\operatorname{scl}(\mathrm{A}) \subseteq \mathrm{U}$ whenever $\mathrm{A} \subseteq \mathrm{U}$ and $\mathrm{U}$ is open in $\mathrm{X}$.

7. A weekly generalized closed ( briefly wg - closed) [13] if $\operatorname{cl}(\operatorname{int}(\mathrm{A})) \subseteq \mathrm{U}$ whenever $\mathrm{A} \subseteq \mathrm{U}$ and $\mathrm{U}$ is open in $\mathrm{X}$.

8. A generalized pre regular closed (gpr closed)[10] if $\operatorname{pcl}(\mathrm{A}) \subseteq \mathrm{U}$ whenever $\mathrm{A} \subseteq \mathrm{U}$ and $\mathrm{U}$ is regular open.

9. A g* closed [26] if $\operatorname{cl}(\mathrm{A}) \subseteq \mathrm{U}$ whenever $\mathrm{A} \subseteq \mathrm{U}$ and $\mathrm{U}$ is g-open.

10. A regular weakly generalized semi closed (rwg closed) [18] if $\mathrm{cl}(\operatorname{int}(\mathrm{A})) \subseteq \mathrm{U}$ whenever $\mathrm{A} \subseteq \mathrm{U}$ and $\mathrm{U}$ is regular open.

11. A $\mathrm{g}^{* *} \operatorname{closed}[21]$ if $\operatorname{cl}(\mathrm{A}) \subseteq \mathrm{U}$ whenever $\mathrm{A} \subseteq \mathrm{U}$ and $\mathrm{U}$ is $\mathrm{g}^{*}$-open.

12. A g\# closed [25] if $\operatorname{cl}(\mathrm{A}) \subseteq \mathrm{U}$ whenever $\mathrm{A} \subseteq \mathrm{U}$ and $\mathrm{U}$ is $\alpha$ g open.

13. A generalized semi-preclosed closed (gsp) closed)[9] if $\operatorname{spcl}(\mathrm{A}) \subseteq \mathrm{U}$ whenever $\mathrm{A} \subseteq \mathrm{U}$ and $\mathrm{U}$ is open.

14. A generalized semi-preclosed star closed ((gsp)* closed)[20] if $\operatorname{cl}(\mathrm{A}) \subseteq \mathrm{U}$ whenever $\mathrm{A} \subseteq \mathrm{U}$ and $\mathrm{U}$ is gsp open.

15. A generalized preclosed closed (gp) closed [14 ] if $\operatorname{pcl}(\mathrm{A}) \subseteq \mathrm{U}$ whenever $\mathrm{A} \subseteq \mathrm{U}$ and $\mathrm{U}$ is open.

16. A gp* closed [11] if $\operatorname{cl}(\mathrm{A}) \subseteq \mathrm{U}$ whenever $\mathrm{A} \subseteq \mathrm{U}$ and $\mathrm{U}$ is gp-open.

17. A regular^ ${ }^{\wedge}$ generalized closed $\left(\mathrm{r}^{\wedge} \mathrm{g}\right.$ closed)[23] if $\operatorname{gcl}(\mathrm{A})$ $\subseteq \mathrm{U}$ whenever $\mathrm{A} \subseteq \mathrm{U}$ and $\mathrm{U}$ is regular open.

18. A regular generalized b-closed (rgb closed) [15] if $\operatorname{bcl}(\mathrm{A}) \subseteq \mathrm{U}$ whenever $\mathrm{A} \subseteq \mathrm{U}$ and $\mathrm{U}$ is regular open.

19. A mildly generalized closed (mildly g closed) [17] if $\operatorname{cl}(\operatorname{int}(\mathrm{A})) \subseteq \mathrm{U}$ whenever $\mathrm{A} \subseteq \mathrm{U}$ and $\mathrm{U}$ is g-open.

20. A r*g*closed set [16] if $\operatorname{rcl}(\mathrm{A}) \subseteq \mathrm{U}$ whenever $\mathrm{A} \subseteq \mathrm{U}$ and $\mathrm{U}$ is $\mathrm{g}$ - open. 


\section{Basic Properties of $\left(r^{*} g^{*}\right) *$-Closed Sets}

Definition 3.1: A subset A of a topological space $(X, \tau)$ is called a $\left(\mathrm{r}^{*} \mathrm{~g}^{*}\right)^{*}$-closed set if $\mathrm{cl}(\mathrm{A}) \subseteq \mathrm{U}$ whenever $\mathrm{A} \subseteq \mathrm{U}$ and $\mathrm{U}$ is $\mathrm{r}^{*} \mathrm{~g}^{*}$-open.

\section{Example 3.2:}

Let $\quad \mathrm{X}=\{\mathrm{a}, \mathrm{b}, \mathrm{c}\}, \quad \tau=\{\phi, \mathrm{X},\{\mathrm{a}\},\{\mathrm{b}\},\{\mathrm{a}, \mathrm{b}\}\}$

Closed sets $=\{\phi, X,\{b, c\},\{a, c\},\{c\}\}$

$\mathrm{r}^{*} \mathrm{~g} *$ open sets of $\mathrm{X}$ are $\phi, \mathrm{X},\{\mathrm{a}\},\{\mathrm{b}\},\{\mathrm{a}, \mathrm{b}\}$

$\left(\mathrm{r}^{*} \mathrm{~g}^{*}\right)^{*}$ closed set are $\{\phi, \mathrm{X},\{\mathrm{c}\},\{\mathrm{b}, \mathrm{c}\},\{\mathrm{a}, \mathrm{c}\}\}$

\section{Proposition 3.3}

Every closed set is $\left(\mathrm{r}^{*} \mathrm{~g} *\right)^{*}$-closed.

\section{Proof:}

Let $\mathrm{A}$ be any closed set in $\mathrm{X}$

To prove : A is $\left(\mathrm{r}^{*} \mathrm{~g}^{*}\right) *$ - closed set

Let $\mathrm{A} \subseteq U$ and $\mathrm{U}$ be any $\mathrm{r}^{*} \mathrm{~g} *$-open set in $\mathrm{X}$

$A$ is closed $\Rightarrow \operatorname{cl}(\mathrm{A})=\mathrm{A} \subseteq U$

$\Rightarrow \operatorname{cl}(\mathrm{A}) \subseteq U$

$\Rightarrow A$ is $\left(\mathrm{r}^{*} \mathrm{~g}^{*}\right)^{*}$-closed set in $\mathrm{X}$.

Hence every closed set is $\left(\mathrm{r}^{*} \mathrm{~g} *\right)^{*}$-closed.

The converse is not true as seen from the following example.

Example 3.4 Let $X=\{a, b, c\}, \tau=\{\varphi, X\{a\},\{a, c\}\}$.

Here $\{a, b\}$ is $\left(\mathrm{r}^{*} \mathrm{~g} *\right)^{*}$ closed but not closed.

\section{Proposition 3. 5}

Every $\mathrm{g}^{*}$ closed set is $\left(\mathrm{r}^{*} \mathrm{~g} *\right)^{*}$ closed set.

Proof: Let $\mathrm{A} \subseteq U$ Where $U$ is $\mathrm{r}^{*} \mathrm{~g}^{*}$ open. Since every $\mathrm{r}^{*} \mathrm{~g} *$ open set is $\mathrm{g}$ open set we have $\operatorname{cl}(\mathrm{A}) \subseteq U$. Therefore $\operatorname{cl}(\mathrm{A}) \subseteq U$ where $\mathrm{U}$ is $\mathrm{r}^{*} \mathrm{~g} *$ open.Hence $\mathrm{A}$ is $\left(\mathrm{r}^{*} \mathrm{~g} *\right)^{*}$ closed.

The converse is not true as seen from the following example.

Example 3.6 Let $X=\{a, b, c\}, \tau=\{\varphi, X\{a\},\{b, c\}\}$.

Here $\{b\}$ is $\left(r^{*} g^{*}\right) *$ closed but not a $g^{*}$ closed.

\section{Proposition 3.7}

Proof:

Every $\left(\mathrm{r}^{*} \mathrm{~g}^{*}\right)^{*}$-closed set is $\mathrm{rg}$-closed

Let $\mathrm{A}$ be any $\left(\mathrm{r}^{*} \mathrm{~g}\right)^{*}$-closed set in $\mathrm{X}$

To prove : A is rg -closed set

Let $\mathrm{A} \subseteq U$ and $\mathrm{U}$ be a regular-open set in $\mathrm{X}$.

Since every regular open set is $\mathrm{r}^{*} \mathrm{~g} *$ open we have $\operatorname{cl}(\mathrm{A}) \subseteq U$ $\Rightarrow \operatorname{cl}(\mathrm{A}) \subseteq U$ whenever $\mathrm{A} \subseteq U$ and $\mathrm{U}$ is regular open in $\mathrm{X}$

$\Rightarrow A$ is $r g$-closed set in $\mathrm{X}$

Hence every $\left(\mathrm{r}^{*} \mathrm{~g}^{*}\right) *$ closed set is $\mathrm{rg}$-closed.

\section{Example 3.8}

The converse need not be true as seen from the following example

Let $\mathrm{X}=\{\mathrm{a}, \mathrm{b}, \mathrm{c}\}$ and $\tau=\{\mathrm{X}, \phi,\{\mathrm{a}, \mathrm{b}\},\{\mathrm{a}\},\{\mathrm{b}\}\}$

Here $\{a . b\}$ is rg closed but not $\left(\mathrm{r}^{*} \mathrm{~g} *\right)^{*}$ closed.

\section{Proposition 3.9}

Every $\left(\mathrm{r}^{*} \mathrm{~g}^{*}\right)^{*}$-closed set is gpr closed.

\section{Proof:}

Let $\mathrm{A} \subseteq U$ and $U$ be an regular open set in X. Since every regular open set is $\mathrm{r}^{*} \mathrm{~g} *$ open we have $\operatorname{cl}(\mathrm{A}) \subseteq \mathrm{U}$

But $\operatorname{pcl}(\mathrm{A}) \subseteq \operatorname{cl}(\mathrm{A}) \subseteq \mathrm{U}$

$\Rightarrow \operatorname{pcl}(\mathrm{A}) \subseteq U$ whenever $\mathrm{A} \subseteq U$ and $\mathrm{U}$ is regular open in $\mathrm{X}$ which implyies Ais gpr closed.

Hence every $\left(\mathrm{r}^{*} \mathrm{~g}^{*}\right) *$ closed set is gpr -closed.

\section{Example 3.10}

The converse need not be true as seen from the following example.

Let $\mathrm{X}=\{\mathrm{a}, \mathrm{b}, \mathrm{c}\}$ and $\tau=\{\mathrm{X}, \phi,\{\mathrm{a}, \mathrm{b}\}\}$

Here $\{\mathrm{a}\}$ is gpr closed but not $\left(\mathrm{r}^{*} \mathrm{~g}^{*}\right)^{*}$ closed .

Proposition 3.11:

Every $\left(\mathrm{r}^{*} \mathrm{~g}^{*}\right)^{*}$-closed set is rwg-closed.

Proof:

Let $A \subseteq U$ and $U$ be an regular open. Since every regular open set is $\mathrm{r}^{*} \mathrm{~g}^{*}$ open we have $\operatorname{cl}(\mathrm{A}) \subseteq \mathrm{U}$

But $\operatorname{cl}(\operatorname{int}(\mathrm{A})) \subseteq \mathrm{cl}(\mathrm{A}) \subseteq \mathrm{U}$

$\Rightarrow \operatorname{cl}(\operatorname{int}(\mathrm{A})) \subseteq U$ whenever $\mathrm{A} \subseteq U$ and $\mathrm{U}$ is regular open in $\mathrm{X}$

Hence A is rwg-closed set in $\mathrm{X}$.

Hence every $\left(\mathrm{r}^{*} \mathrm{~g} *\right)^{*}$ closed set is rwg-closed.

Example:3.12

The converse need not be true as seen from the following example.

Let $\mathrm{X}=\{\mathrm{a}, \mathrm{b}, \mathrm{c}\}, \tau=\{\mathrm{X}, \phi,\{\mathrm{a}\}\}$

Here $\{\mathrm{a}, \mathrm{b}\}$ is $\left(\mathrm{r}^{*} \mathrm{~g} *\right)^{*}$ closed but not rwg closed

\section{Proposition 3.13:}

Every $\mathrm{g}^{* *}$ closed set is $\left.\left(\mathrm{r}^{*} \mathrm{~g}\right)^{*}\right)^{*}$-closed set.

Proof: Let $A \subseteq U$ and $U$ be an $r^{*} g^{*}$ open set in $X$. Since every $\mathrm{r}^{*} \mathrm{~g} *$ open set is $\mathrm{g}^{*}$ open we have $\mathrm{cl}(\mathrm{A}) \subseteq \mathrm{U}$.Therefore $\mathrm{cl}(\mathrm{A}) \subseteq \mathrm{U}$ where $\mathrm{U}$ is $\mathrm{r}^{*} \mathrm{~g}^{*}$ open. Hence A is $\left(\mathrm{r}^{*} \mathrm{~g}^{*}\right)^{*}$ closed.

\section{Example:3.14}

The converse need not be true as seen from the following example.

Let $\mathrm{X}=\{\mathrm{a}, \mathrm{b}, \mathrm{c}\}, \tau=\{\mathrm{X}, \phi,\{\mathrm{a}\},\{\mathrm{a}, \mathrm{c}\}\}$

Here $\{\mathrm{a}\}$ is $\left(\mathrm{r}^{*} \mathrm{~g} *\right)^{*}$ closed but not $\mathrm{g}^{* *}$ closed

\section{Proposition 3.15:}

Every g\# closed set is $\left(\mathrm{r}^{*} \mathrm{~g} *\right)^{*}$-closed set .

Proof:

Let $A \subseteq U$ and $U$ be an $r^{*} g^{*}$ open set in $X$. Since every $r^{*} g^{*}$ open set is $\alpha g$ open we have $\operatorname{cl}(\mathrm{A}) \subseteq \mathrm{U}$. Hence $\operatorname{cl}(\mathrm{A}) \subseteq \mathrm{U}$ where $\mathrm{U}$ is $\mathrm{r}^{*} \mathrm{~g}$ *open.

Hence every g\# closed set is $\left(\mathrm{r}^{*} \mathrm{~g} *\right)^{*}$ closed.

\section{Example:3.16}

The converse need not be true as seen from the following example

\section{Volume 4 Issue 12, December 2015}




\section{International Journal of Science and Research (IJSR) \\ ISSN (Online): 2319-7064}

Index Copernicus Value (2013): 6.14 | Impact Factor (2014): 5.611

Let $\mathrm{X}=\{\mathrm{a}, \mathrm{b}, \mathrm{c}\}, \tau=\{\mathrm{X}, \phi,\{\mathrm{a}\},\{\mathrm{b}, \mathrm{c}\}\}$

Here $\{b\}$ is $\left(r^{*} g^{*}\right)^{*}$ closed but not $g \#$ closed.

\section{Proposition 3.17:}

Every (gsp)* closed set is $\left(\mathrm{r}^{*} \mathrm{~g}^{*}\right)^{*}$-closed.

\section{Proof:}

Proof follows from the definition of (gsp)* closed set and fact that $\mathrm{r}^{*} \mathrm{~g}^{*}$ open implies gsp open.

\section{Example:3.18}

The converse need not be true as seen from the following example

Let $X=\{a, b, c\}, \tau=\{X, \phi,\{b\},\{a, c\}\}$

Here $\{\mathrm{a}, \mathrm{c}\}$ is not $(\mathrm{gsp})^{*}$ closed but $\left(\mathrm{r}^{*} \mathrm{~g} *\right)^{*}$ closed

Proposition 3.19: Every gp* closed set is $\left(\mathrm{r}^{*} \mathrm{~g}^{*}\right)^{*}$-closed.

Proof: Proof follows from the definition of $(\mathrm{gp}) *$ closed set and fact that $\mathrm{r}^{*} \mathrm{~g}$ *open implies gp open

\section{Example:3.20}

The converse need not be true as seen from the following example

$\mathrm{X}=\{\mathrm{a}, \mathrm{b}, \mathrm{c}\}, \tau=\{\mathrm{X}, \phi,\{\mathrm{c}\},\{\mathrm{a}, \mathrm{c}\}\}$

Here $\{\mathrm{a}, \mathrm{c}\}$ is not $(\mathrm{gp})^{*}$ closed but $\left(\mathrm{r}^{*} \mathrm{~g}^{*}\right)^{*}$ closed .

Proposition 3.21: Every $\left(\mathrm{r}^{*} \mathrm{~g}^{*}\right)^{*}$ closed set is $\mathrm{r}^{\wedge} \mathrm{g}$-closed.
Let $\mathrm{A} \subseteq U$ and $U$ be an regular open set in $X$. Since every regular open set is $\mathrm{r}^{*} \mathrm{~g} *$ open we have $\operatorname{cl}(\mathrm{A}) \subseteq \mathrm{U}$

But $\operatorname{gcl}(\mathrm{A}) \subseteq \operatorname{cl}(\mathrm{A}) \subseteq \mathrm{U}$

$$
\Rightarrow \operatorname{gcl}(\mathrm{A}) \subseteq U \text { whenever } \mathrm{A} \subseteq U \text { and } \mathrm{U} \text { is regular open }
$$
in $\mathrm{X}$

Which implsies $A$ is $\mathrm{r}^{\wedge} \mathrm{g}$ closed.

Hence every $\left(\mathrm{r}^{*} \mathrm{~g} *\right)^{*}$ closed set is $\mathrm{r}^{\wedge} \mathrm{g}$-closed

\section{Example:3.22}

The converse need not be true as seen from the following example

$\mathrm{X}=\{\mathrm{a}, \mathrm{b}, \mathrm{c}\}, \tau=\{\mathrm{X}, \phi,\{\mathrm{a}\},\{\mathrm{b}\},\{\mathrm{a}, \mathrm{c}\}\}$

Here $\{\mathrm{a}, \mathrm{b}\}$ is $\left(\mathrm{r}^{\wedge} \mathrm{g}\right)$ closed but not $\left(\mathrm{r}^{*} \mathrm{~g}^{*}\right)^{*}$ closed .

Proposition 3.23: Every $\left(\mathrm{r}^{*} \mathrm{~g}^{*}\right)^{*}$ closed set is rgb-closed. Proof:

Let $\mathrm{A} \subseteq U$ and $U$ be an regular open set in $\mathrm{X}$. Since every regular open set is $\mathrm{r}^{*} \mathrm{~g} *$ open we have $\operatorname{cl}(\mathrm{A}) \subseteq \mathrm{U}$

But $\mathrm{bcl}(\mathrm{A}) \subseteq \mathrm{cl}(\mathrm{A}) \subseteq \mathrm{U}$

$\Rightarrow \operatorname{bcl}(\mathrm{A}) \subseteq U$ whenever $\mathrm{A} \subseteq U$ and $\mathrm{U}$ is regular open in X Which implies $\mathrm{A}$ is rgb closed.

\section{Example:3.24}

The converse need not be true as seen from the following example

Let $\mathrm{X}=\{\mathrm{a}, \mathrm{b}, \mathrm{c}\}, \tau=\{\mathrm{X}, \phi,\{\mathrm{c}\}\}$

Here $\{b\}$ is rgb closed but not $\left(\mathrm{r}^{*} \mathrm{~g}^{*}\right)^{*}$ closed.

Thus We have the following diagram.

\section{Proof:}

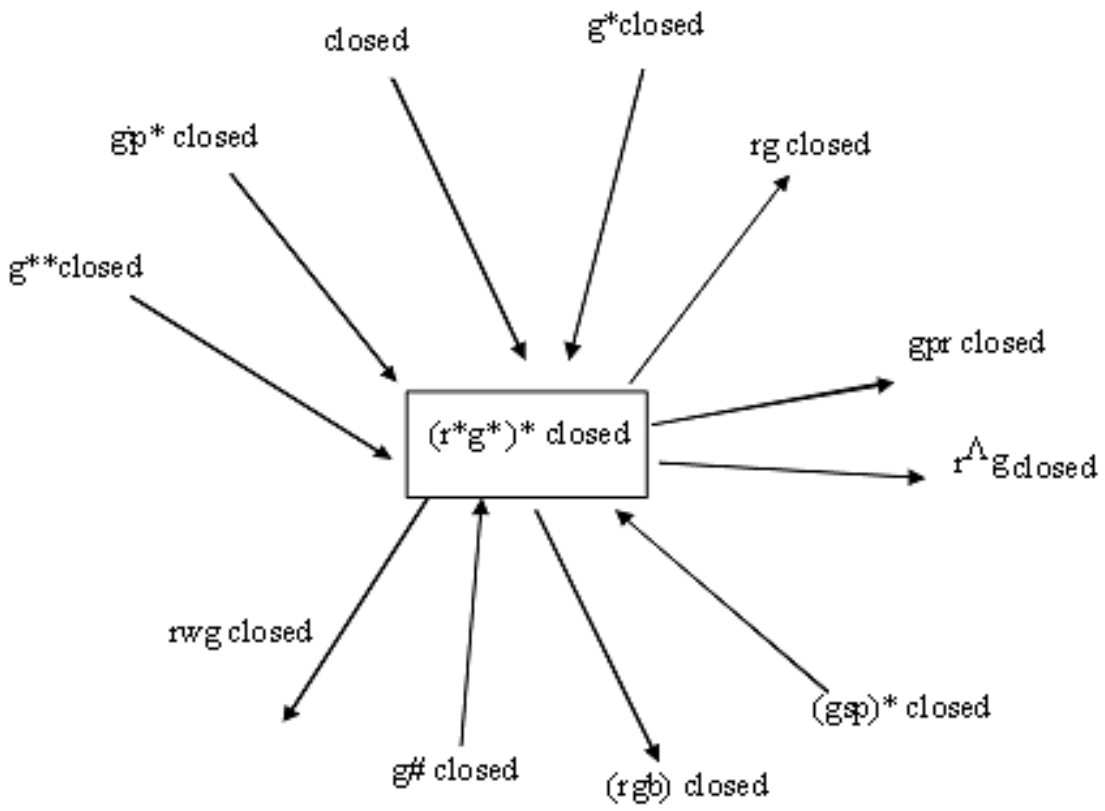

Here $\mathrm{A} \rightarrow \mathrm{B}$ represents $\mathrm{A}$ implies $\mathrm{B}$. But not conversely

\section{Remark:3.25}

$\left(\mathrm{r}^{*} \mathrm{~g}^{*}\right)^{*}$ closed sets and semi closed sets are independent of each other as seen from the following examples.

\section{Example:3.26}

Let $\mathrm{X}=\{\mathrm{a}, \mathrm{b}, \mathrm{c}\}, \tau=\{\mathrm{X}, \phi,\{\mathrm{a}\},\{\mathrm{b}\},\{\mathrm{a}, \mathrm{b}\}\}$.Here $\{\mathrm{b}\}$ is semi closed but not $\left(\mathrm{r}^{*} \mathrm{~g}^{*}\right)^{*}$ closed
Let $\mathrm{X}=\{\mathrm{a}, \mathrm{b}, \mathrm{c}\} . \tau=\{\mathrm{X}, \phi,\{\mathrm{a}\},\{\mathrm{a}, \mathrm{c}\}\}$.Here $\{\mathrm{a}, \mathrm{b}\}$ is $\left(\mathrm{r}^{*} \mathrm{~g}^{*}\right)^{*}$ closed but not semi closed

Remark: 3.27

$\left(\mathrm{r}^{*} \mathrm{~g} *\right)^{*}$ closed sets and semi pre closed sets are independent of each other as seen from the following examples. 


\section{International Journal of Science and Research (IJSR) \\ ISSN (Online): 2319-7064}

Index Copernicus Value (2013): 6.14 | Impact Factor (2014): 5.611

\section{Example :3.28}

Let $\mathrm{X}=\{\mathrm{a}, \mathrm{b}, \mathrm{c}\}, \tau=\{\mathrm{X}, \phi,\{\mathrm{a}\},\{\mathrm{b}\},\{\mathrm{a}, \mathrm{b}\}\}$. Here $\{\mathrm{a}\}$ is semi pre closed but not $\left(\mathrm{r}^{*} \mathrm{~g} *\right)^{*}$ closed.

Example 3.29: Let $\mathrm{X}=\{\mathrm{a}, \mathrm{b}, \mathrm{c}\}, \tau=\{\mathrm{X}, \phi,\{\mathrm{a}\},\{\mathrm{a}, \mathrm{c}\}\}$.Here $\{b\}$ is $\left(r^{*} g^{*}\right) *$ closed but not semi pre closed.

\section{Remark: 3.30}

The following example shows that $\left(\mathrm{r}^{*} \mathrm{~g} *\right)^{*}$ closedness is independent from sg closedness, gs closedness, pre closedness and wg closedness

\section{Example 3.31}

Let $\mathrm{X}=\{\mathrm{a}, \mathrm{b}, \mathrm{c}\}, \tau=\{\mathrm{X}, \phi,\{\mathrm{a}\},\{\mathrm{a}, \mathrm{c}\}\}$

(i) $\quad\{\mathrm{c}\}$ is sg closed but not $\left(\mathrm{r}^{*} \mathrm{~g}^{*}\right)^{*}$ closed.$\{\mathrm{a}\}$ is $\left(\mathrm{r}^{*} \mathrm{~g}\right)^{*}$ closed but not sg closed .

(ii) $\quad\{\mathrm{c}\}$ is gs closed but not $\left(\mathrm{r}^{*} \mathrm{~g}^{*}\right)^{*}$ closed. $\{\mathrm{a}\}$ is $\left(\mathrm{r}^{*} \mathrm{~g}^{*}\right)^{*}$ closed but not gs closed.

(iii) $\{\mathrm{c}\}$ is pre closed but not $\left(\mathrm{r}^{*} \mathrm{~g}^{*}\right)^{*}$ closed. $\{\mathrm{a}\}$ is $\left(\mathrm{r}^{*} \mathrm{~g} *\right)^{*}$ closed but not pre closed .

(iv) $\{c\}$ is wg closed but not $\left(\mathrm{r}^{*} \mathrm{~g}^{*}\right)^{*}$ closed. $\{\mathrm{a}\}$ is $\left(\mathrm{r}^{*} \mathrm{~g}^{*}\right)^{*}$ closed but not wg closed

\section{Remark:3.32}

$\left(\mathrm{r}^{*} \mathrm{~g} *\right)^{*}$ closed sets and $\alpha$-closed sets are independent of each other as seen from the follwing examples.

\section{Example:3.33}

Let $\mathrm{X}=\{\mathrm{a}, \mathrm{b}, \mathrm{c}\}, \tau=\{\mathrm{X}, \phi,\{\mathrm{a}\},\{\mathrm{b}\},\{\mathrm{a}, \mathrm{b}\}\}$. Here $\{\mathrm{c}\}$ is $\left(\mathrm{r}^{*} \mathrm{~g}^{*}\right)^{*}$ closed but not $\alpha$-closed .

Let $\mathrm{X}=\{\mathrm{a}, \mathrm{b}, \mathrm{c}\}, \tau=\{\mathrm{X}, \phi,\{\mathrm{b}\},\{\mathrm{a}, \mathrm{b}\}\}$. Here $\{\mathrm{a}\}$ is $\alpha$-closed but not $\left(\mathrm{r}^{*} \mathrm{~g}^{*}\right)^{*}$ closed.

Thus we have the following diagram

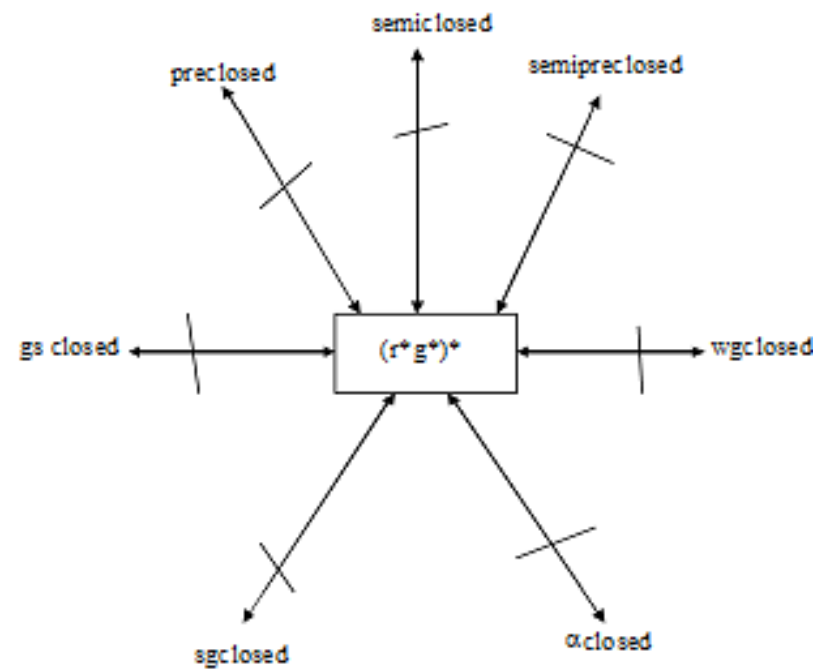
A $\nrightarrow$
$\mathrm{B}$ represents $\mathrm{A}$ and $\mathrm{B}$ are independent of Each other.

\section{Theorem:3.34}

The union of two $\left(\mathrm{r}^{*} \mathrm{~g} *\right)^{*}$-closed sets is $\left(\mathrm{r}^{*} \mathrm{~g} *\right)^{*}$-closed set

\section{Proof:}

let $\mathrm{A}$ and $\mathrm{B}$ be $\left(\mathrm{r}^{*} \mathrm{~g}^{*}\right)^{*}$-closed sets in $\mathrm{X}$ and $\mathrm{U}$ be any $\mathrm{r}^{*} \mathrm{~g}{ }^{*}$ open set containing $\mathrm{A}$ and $\mathrm{B}$ such that , $\mathrm{cl}(\mathrm{A}) \subseteq \mathrm{U}$, whenever $\mathrm{A} \subseteq U$ and $\mathrm{U}$ is $\mathrm{r}^{*} \mathrm{~g}^{*}$-open and $\mathrm{cl}(\mathrm{B}) \subseteq \mathrm{U}$, whenever $\mathrm{B} \subseteq U$ and $\mathrm{U}$ is $\mathrm{r}^{*} \mathrm{~g}^{*}$-open .Let $\mathrm{A} \cup B \subseteq U$. Then $\mathrm{A} \subseteq U$ and $\mathrm{B} \subseteq U$.

Hence $\operatorname{cl}(\mathrm{A} \cup B)=\operatorname{cl}(\mathrm{A}) \cup \operatorname{cl}(\mathrm{B}) \subseteq U$

$\Rightarrow \operatorname{cl}(\mathrm{AU} B) \subseteq U$ whenever $\mathrm{AUB} \subseteq \mathrm{U}$ and $\mathrm{U}$ is $\left(\mathrm{r}^{*} \mathrm{~g}^{*}\right)$ open set in $\mathrm{X}$.

Hence AUB is $\left(\mathrm{r}^{*} \mathrm{~g} *\right)^{*}$ closed.

Remark: 3.35 The intersection of two $\left(\mathrm{r}^{*} \mathrm{~g}^{*}\right)^{*}$ closed sets need not be $\left(\mathrm{r}^{*} \mathrm{~g}^{*}\right) *$ closed.

Let $\mathrm{X}=\{\mathrm{a}, \mathrm{b}, \mathrm{c}\} \quad \tau=\{\varphi, \mathrm{X},\{\mathrm{a}\}\} .\left(\mathrm{r}^{*} \mathrm{~g} *\right)^{*} \quad$ closed sets are $\{\varphi, X,\{b\},\{c\},\{a, b\},\{b, c\},\{a, c\}\}$. The intersection of $\{a, b\}$ and $\{\mathrm{a}, \mathrm{c}\}$ is not $\mathrm{a}\left(\mathrm{r}^{*} \mathrm{~g} *\right)^{*}$ closed set.

\section{Proposition 3.36:}

If $\mathrm{A}$ is both $\left(\mathrm{r}^{*} \mathrm{~g} *\right)^{*}$-open and $\left(\mathrm{r}^{*} \mathrm{~g} *\right)^{*}$-closed, then $\mathrm{A}$ is closed.

\section{Proof:}

Let $\mathrm{A}$ be $\mathrm{r}^{*} \mathrm{~g} *$-open set in $\mathrm{X}$ and also ( $\mathrm{r}^{*} \mathrm{~g} *$ )-closed set in $\mathrm{X}$ $\mathrm{Cl}(\mathrm{A})) \subseteq U$ whenever $\mathrm{A} \subseteq \mathrm{U}$ and $\mathrm{U}$ is $\left(\mathrm{r}^{*} \mathrm{~g}^{*}\right)$-open set in $\mathrm{X}$ Since $\mathrm{A}$ is $\mathrm{r}^{*} \mathrm{~g} *$-open. Take $\mathrm{U}=\mathrm{A} \Rightarrow \operatorname{cl}(\mathrm{A}) \subseteq U=\mathrm{A} \Rightarrow$ $\operatorname{cl}(\mathrm{A}) \subseteq A$

But $\mathrm{A} \subseteq \operatorname{cl}(A) \Rightarrow \mathrm{Cl}(\mathrm{A})=\mathrm{A}$. Hence $\mathrm{A}$ is closed.

Proposition:3.37 Let A be a $\left(\mathrm{r}^{*} \mathrm{~g}^{*}\right)^{*}$ closed set of $(\mathrm{X}, \tau)$. Then cl(A)-A does not contain any non empty $\mathrm{r}^{*} \mathrm{~g}^{*}$ closed set.

Proof: Let $\mathrm{F}$ be a $\mathrm{r}^{*} \mathrm{~g} *$ closed set such that $\mathrm{F} \subseteq \operatorname{cl}(\mathrm{A})$ A.Then $\mathrm{A} \subseteq \mathrm{X}$-F.Since $\mathrm{A}$ is $\left(\mathrm{r}^{*} \mathrm{~g} *\right)^{*}$ closed andX-F is $\mathrm{r}^{*} \mathrm{~g} *$ open we have $\mathrm{cl}(\mathrm{A}) \subseteq \mathrm{X}-\mathrm{F}$. This implies $\mathrm{F} \subseteq \mathrm{X}$ cl(A).So $\mathrm{F} \subseteq(\mathrm{X}-\mathrm{cl}(\mathrm{A})) \cap(\mathrm{cl}(\mathrm{A})-\mathrm{A}) \subseteq(\mathrm{X}-\mathrm{cl}(\mathrm{A})) \cap \mathrm{cl}(\mathrm{A})$ which implies $\mathrm{F}=\varphi$.Hence the theorem.

Proposition: 3.38 If A is mildly g closed and open then A is $\left(\mathrm{r}^{*} \mathrm{~g}\right)^{*}$ closed.

Proof: Let $A \subseteq U$ and $U$ is $\mathrm{r}^{*} \mathrm{~g}^{*}$-open set.But every $\mathrm{r}^{*} \mathrm{~g} *$ open is g-open, We have $\operatorname{cl}(\operatorname{int}(\mathrm{A})) \subseteq \mathrm{U}$ which implies $\mathrm{cl}(\mathrm{A}) \subseteq \mathrm{U}$ where Uis $\mathrm{r}^{*} \mathrm{~g}{ }^{*}$ open. Hence Ais $\left(\mathrm{r}^{*} \mathrm{~g}\right)^{*}$ )closed.

\section{Proposition 3.39:}

If $A$ is $\left(r^{*} g^{*}\right)^{*}$ - closed set of $(X, \tau)$, such that $A$ $\subseteq \mathrm{B} \subseteq \mathrm{cl}(\mathrm{A})$, then $\mathrm{B}$ is also a $\left(\mathrm{r}^{*} \mathrm{~g}^{*}\right)^{*}$-closed set of $(\mathrm{X}, \tau)$.

Proof:

Given that $\mathrm{A}$ is $\left(\mathrm{r}^{*} \mathrm{~g} *\right)^{*}$ - closed set of $(\mathrm{X}, \tau)$. $\Rightarrow \operatorname{cl}(\mathrm{A}) \subseteq \mathrm{A}$ whenever $\mathrm{A} \subseteq \mathrm{U}$ and $\mathrm{U}$ is $\mathrm{r}^{*} \mathrm{~g}^{*}$ -

open set in $\mathrm{X}$ 


\section{International Journal of Science and Research (IJSR) \\ ISSN (Online): 2319-7064}

Index Copernicus Value (2013): 6.14 | Impact Factor (2014): 5.611

To prove : $\mathrm{B}$ is $\left(\mathrm{r}^{*} \mathrm{~g}^{*}\right)^{*}$-closed set .

$$
\begin{aligned}
\text { Let } \mathrm{B} \subseteq \mathrm{U} \text {. Now } \operatorname{cl}(\mathrm{B}) \subseteq \operatorname{cl}(\mathrm{A}) \subseteq \mathrm{U} \\
\Rightarrow \operatorname{cl}(\mathrm{B}) \subseteq \mathrm{U} \text { whenever } \mathrm{B} \subseteq \mathrm{U} \text { and } \mathrm{U} \text { is }
\end{aligned}
$$

$\left(\mathrm{r}^{*} \mathrm{~g} *\right)^{*}$ open..

$\Rightarrow \mathrm{B}$ is $\left(\mathrm{r}^{*} \mathrm{~g}^{*}\right)^{*}$-closed

\section{4. $\left(\mathrm{r}^{*} \mathrm{~g} *\right)^{*}$ Open Sets}

Definition: 4.1 A set $\mathrm{A} \subseteq \mathrm{X}$ is called( $\left.\mathrm{r}^{*} \mathrm{~g}^{*}\right)^{*}$ open set if its complement is $\left(\mathrm{r}^{*} \mathrm{~g}^{*}\right)^{*}$ closed.

Theorem: 4.2 A subset $\mathrm{A} \subseteq \mathrm{X}$ is $\left(\mathrm{r}^{*} \mathrm{~g}^{*}\right)^{*}$ open iff there exists a ${ }^{*} \mathrm{~g}^{*}$ closed set $\mathrm{F}$ such that $\mathrm{F} \subseteq$ int $\mathrm{A}$ whenever $\mathrm{F}$ $\subseteq \mathrm{A}$

Proof : Let $\mathrm{A}$ be a $\left(\mathrm{r}^{*} \mathrm{~g}^{*}\right)^{*}$ closed and $\mathrm{F} \subseteq \mathrm{A}$ then $\mathrm{X}-\mathrm{A}$ $\subseteq \mathrm{X}-\mathrm{F}$ where $\mathrm{X}-\mathrm{F}$ is $\left(\mathrm{r}^{*} \mathrm{~g}^{*}\right)^{*}$ open .Therefore

$\mathrm{Cl}(\mathrm{X}-\mathrm{A}) \quad \subseteq \mathrm{X}-\mathrm{F}$ which impliesX $-($ intA $) \subseteq \mathrm{X}-\mathrm{F}$

Therefore we have $\mathrm{F} \subseteq$ int (A)

Conversely suppose $\mathrm{F} \subseteq$ int $\mathrm{A}$ where $\mathrm{F}$ is $\left(\mathrm{r}^{*} \mathrm{~g}^{*}\right)$ closed $\mathrm{F}$ $\subseteq \mathrm{A}$

Let $\mathrm{X}-\mathrm{A} \subseteq \mathrm{U}$ where is $\mathrm{U}$ is $\left(\mathrm{r}^{*} \mathrm{~g}^{*}\right) *$ open

Then $\mathrm{X}-\mathrm{U} \subseteq \mathrm{A}$ where $\mathrm{X}-\mathrm{U}$ is $\left(\mathrm{r}^{*} \mathrm{~g} *\right)^{*}$ closed by hypothesis $\mathrm{X}-\mathrm{U} \subseteq$ int A which implies $\mathrm{X}$ - int (A) $\subseteq \mathrm{U}$ Which implies $\mathrm{Cl}(\mathrm{X}-\mathrm{A}) \subseteq \mathrm{U}$ where $\mathrm{U}$ is $\left(\mathrm{r}^{*} \mathrm{~g} *\right)^{*}$ open and hence $\mathrm{X}-\mathrm{A}$ is $\left(\mathrm{r}^{*} \mathrm{~g}^{*}\right)^{*}$ closed.

Hence $\mathrm{A}$ is $\left(\mathrm{r}^{*} \mathrm{~g}^{*}\right)^{*}$ open.

Theorem 4.3: If int $\mathrm{A} \subseteq \mathrm{B} \subseteq \mathrm{A}$ and if $\mathrm{A}$ is $\left(\mathrm{r}^{*} \mathrm{~g}^{*}\right)^{*}$ open then $\mathrm{B}$ is $\left(\mathrm{r}^{*} \mathrm{~g} *\right)^{*}$ open

Proof: $\mathrm{B} \subseteq \mathrm{A}$ implies $\mathrm{X}-\mathrm{A} \subseteq \mathrm{X}-\mathrm{B}$

Int $\mathrm{A} \subseteq \mathrm{B}$ implies $\mathrm{X}-\mathrm{B} \subseteq \mathrm{X}$ - int $\mathrm{A}$

That is $\mathrm{X}-\mathrm{A} \subseteq \mathrm{X}-\mathrm{B} \subseteq \mathrm{X}-\operatorname{int}(\mathrm{A})=\mathrm{Cl}(\mathrm{X}-\mathrm{A})$

Since $\mathrm{X}-\mathrm{A}$ is $\left(\mathrm{r}^{*} \mathrm{~g}^{*}\right)^{*}$ closed, by theorem (3.39) $\mathrm{X}-\mathrm{B}$ is $\left(\mathrm{r}^{*} \mathrm{~g} *\right)^{*}$ closed which impliesB is $\left(\mathrm{r}^{*} \mathrm{~g}^{*}\right)^{*}$ open.

Theorem4.5 : Let $\mathrm{B} \subseteq \mathrm{X}$. if $\mathrm{B}$ is $\left(\mathrm{r}^{*} \mathrm{~g}^{*}\right)^{*}$ open and int $\mathrm{B}$ $\subseteq \mathrm{A}$ then $\mathrm{A} \cap \mathrm{B}$ is $\left(\mathrm{r}^{*} \mathrm{~g}^{*}\right)^{*}$ open.

Proof : It is given that int $\mathrm{B} \subseteq \mathrm{A}$ and also int $\mathrm{B} \subseteq \mathrm{B}$, implies Int $\mathrm{B} \subseteq \mathrm{A} \cap \mathrm{B} \subseteq \mathrm{B}$. By theorem $3.39 \mathrm{~A} \cap \mathrm{B}$ is $\left(\mathrm{r}^{*} \mathrm{~g}^{*}\right)^{*}$ open.

Theorem 4.6: A set A is $\left(\mathrm{r}^{*} \mathrm{~g}^{*}\right)^{*}$ closed if $\operatorname{cl}(\mathrm{A})-\mathrm{A}$ is $\left(\mathrm{r}^{*} \mathrm{~g} *\right)^{*}$ open

Proof: Let A be ( $\left.\mathrm{r}^{*} \mathrm{~g} *\right)^{*}$ closed

Let $\mathrm{F}$ be a $\mathrm{r}^{*} \mathrm{~g} *$ closed set such that $\mathrm{F} \subseteq \mathrm{cl}(\mathrm{A})-\mathrm{A}$

Then by theorem (3.37) $\mathrm{F}=\varphi$

$\mathrm{F} \subseteq \operatorname{int}(\mathrm{cl}(\mathrm{A})-\mathrm{A})$

$\Rightarrow \quad \mathrm{cl}(\mathrm{A})-\mathrm{A}$ is $\left(\mathrm{r}^{*} \mathrm{~g}^{*}\right)^{*}$ open by Theorem (4.2).

\section{Conclusion}

In this paper we have introduced $\left(\mathrm{r}^{*} \mathrm{~g}^{*}\right) *$ closed sets and $\left(\mathrm{r}^{*} \mathrm{~g} *\right)^{*}$ open sets and studied some properties. This class of sets can be used to discuss the notion of Continuity, Compactness and connectedness and also can be extended to other topological spaces like Fuzzy \&Bitopological Spaces.

\section{References}

[1] M.E.Abd El-Monsef, S.N.El.Deeb and R.A.Mohamoud, $\beta$ open sets and $\beta$ continuous mappings, Bull. Fac.Sci. Assiut Univ.,12(1983), 77-80.

[2] S.P.Arya and T.M.Nour, characterizations of S normal spaces , Indian J.Pure app.Math,21(1990)

[3] D.Andrijevic, Semi-pre opensets, Mat. Vesnik, 38(1)1986, 24-32.

[4] K.Balachandran ,P.Sundaram and H.Maki, On generalized continuous maps in topological spaces, Mem.Fac.Kochi univ.ser.A.Maths., 12(1991)5-13.

[5] S.SBenchelli and R.S.wali, On rw-closed sets in topological spaces , Bull.malayas.math.soc(2007),99110.

[6] P.Bhatacharya and B.K.Lahiri, Semi-generalized closed sets in topology, Indian J.Math, 29(3)(1987),375-382.

[7] N.Biswas, On Characterizations of semi-continuous functions, Atti, Accad. Naz. Lincei Rend. Cl.Fis.Mat. Natur., 48(8) (1970), 399-402.

[8] R.Devi, H.Maki and K.Balachandran, Generalized $\alpha$ closed maps and $\alpha$ generalized closed maps, Indian.J.Pure.Appl.Math, 29(1)(1998),37-49.

[9] J.Dontchev.On Genralizing semi pre open sets, Mem.Fac.Sci.Kochiser.a.Math., 16(1995),35-48

[10] Gnanambal.Y,On generalized pre regular closed sets in Topological Spaces,Indian J.Pure App.Maths,28(1997),351-360.

[11]P.Jayakumar,,K.Mariyappa, S.Sekar, on generalized gp* closed sets in topological spaces et al,int.journal of Math analysis, Vol 7 2013,no 33,1635-1645.

[12] N.Levine, Generalized closed sets in topology, Rend.Circ.Math.Palermo,19(2)(1970),89-96.

[13]H.Maki R.Devi,and K.Balachandran, Associated topologies of generalized $\alpha$ closed and $\alpha$ generalized closed sets , Mem.Fac.Sci.kochi univ.ser.a, math.,15(1994),51-63

[14]H.Maki,J.Umehara and T.Noiri , Every topological space is pre- $\mathrm{T}_{1 / 2}$ Mem.Fac.Sci.kochi univ.ser.a, math.,17(1996),33-42

[15]K.Mariappa and S.Sekar,On Regular Generalised bclosed Set,Int.Journal of Math. Analysis, vol,2013,no.13,613-624.

[16] N.Meenakumari and T.Indira , $\mathrm{r}^{*} \mathrm{~g} *$ closed sets in topological spaces, Annals of Pure and Applied Mathematics vol.6, No. 2,2014,125-132

[17] C.Mugundan,Nagaveni.N, A Weaker form of closed sets, 2011,949-961.

[18] N.Nagaveni,Studies on Generalisation of Homeomorphisms in Topological spaces,Ph.d Thesis, BharathiyarUniversity, Coimbatore.

[19] A.Narmadha \& Nagaveni, On regular b-open sets in Topological spaces, Int.Journal of math.Analysis, Vol.7,2013,no.19,937-948.

[20] M.Pauline Mary Helen and A.Kulandai Therese, (gsp)*closed sets in topological spaces,IJMTT-Volume 6 February 2014,ISSN NO2231-5373.

[21] Pauline Mary Helen.M,Veronica Vijayan,Ponnuthai Selvarani,g** closed sets in Topological Spaces,IJMA 3(5),(2012),1-15.

[22] N.Palaniappan \&K.C.Rao,Regular generalized closed sets ,Kyungpook Math.3(2)(1993),211.

\section{Volume 4 Issue 12, December 2015}




\section{International Journal of Science and Research (IJSR) \\ ISSN (Online): 2319-7064}

Index Copernicus Value (2013): 6.14 | Impact Factor (2014): 5.611

[23] Savithri.D\&Janaki.C,On Regular^ Generalizedclosed sets in Topological spaces,IJMA- 4(4)2013,162-169.

[24] M.Stone,Application of the Boolean rings to general topology, Trans.Amer.Math.Soc.41 (1937), 374-481.

[25] M.K.R.S.Veerakumar, $\mathrm{g}^{\#}$-closed sets in topological spaces,Mem.Fac.Sci.Kochi

Univ Ser.A.,Math.,24(2003),1-13.

[26] M.K.R.S.Veerakumar, Between closed sets and $\mathrm{g}$ closed sets,Mem.Fac.Sci.Koch Univ.Ser.A. Math., 21 (2000) 1-19. 